\title{
Almonertinib in the treatment of lung cancer with breast metastasis: a case report
}

Xuyu Gu ( $\sim$ gu1806@126.com )

Southeast University

Chanchan Gao

Southeast University Zhongda Hospital

Longfei Wang

Southeast University Zhongda Hospital

Shiya Zheng

Southeast University Zhongda Hospital

\section{Case report}

Keywords: Breast metastasis, EGFR 21 exon p.L858R, lung adenocarcinoma

Posted Date: December 15th, 2020

DOI: https://doi.org/10.21203/rs.3.rs-128250/v1

License: (a) This work is licensed under a Creative Commons Attribution 4.0 International License. Read Full License 


\section{Abstract}

\section{Background}

Lung adenocarcinoma with breast metastasis is rare. In the present study, a case of an advanced patient with breast metastasis from lung adenocarcinoma with EGFR 21 exon p.L858R mutation who underwent TKI-inhibitors is reported.

Case presentation

A 62-year-old female patient diagnosed with lung adenocarcinoma who had undergone seven times disease progress and breast metastasis in sixth time disease progress. The patient underwent left breast puncture and axillary lymph node in ultrasound-guided and the postoperative pathological diagnosis of metastatic lung adenocarcinoma was confirmed. And then gene detection showed EGFR 21 exon p.L858R mutation. Breast metastasis for lung adenocarcinoma was diagnosed and the patient are being treated with Almonertinib.

\section{Conclusion}

Breast metastasis is rare and lung adenocarcinoma might be the primary disease. Gene indection is important. And for lung cancer patients with recurrent pleural effusion, visit of the breast should be included in the follow-up process.

\section{Background}

Lung cancer is the most common malignant tumor and the leading cause of cancer-related death in China ${ }^{1}$. Non-small cell lung cancer (NSCLC) accounts for more than $80 \%$ of all lung cancers ${ }^{2}$. Despite the advanced progress made in the treatments of NSCLC, the prognosis is still not satisfactory and the 5-year survival rate is lower than $20 \%^{3}$. The most common sites of metastasis in patients with NSCLC are brain, bone, adrenal gland, and lungs ${ }^{4}$.Lung metastasis of breast cancer is more common in clinical. However, breast metastasis from primary lung cancer is rather rare, about $0.41 \%$ of breast cancer ${ }^{5}$. In this case report, we illustrate a case of lung adenocarcinoma causing breast metastasis and report the clinicopathological features and therapeutics interventions, as well as the outcomes of this aggressive disease. We hope that this case report can provide a reference to avoid misdiagnosis and mistreatment and give patients more reasonable treatment.

\section{Case Presentation}

A 62-year-old Chinese female complained of coughing and chest tightness at the the first visit (in 2017). An enhanced chest computed tomography (CT) was performed and revealed a mass in the left lower lobe and massive pericardiac fluid. The patient underwent pericardiocentesis drainage together with pericardial effusion cytology, which revealed lung adenocarcinoma and EGFR 21 mutation, mutation 
abundance 10.83\%. She then treated with Gefitinib (Iressa, $0.25 \mathrm{~g}$, qd) and cardiac perfusion with Cisplatin (10 mg).

After nine months,the patient developed chest tightness again in March 2018. An enhanced chest CT showed massive pleural effusion, followed by ascites cytology revealing lung adenocarcinoma cells. Then The patient received 5 cycles of systemic chemotherapy with Pemetrexed (Pemetrexed $425 \mathrm{mg} / \mathrm{m}^{2}$, Cis-platinum $64 \mathrm{mg} / \mathrm{m}^{2}$, q3w ) and cis-platinum intrapleural infusion $(20 \mathrm{mg})$. However, the patient refused further chemotherapy and took 6 cycles of Anlotinib (12 mg qd, d1-d14, q3w) by herself. Unfortunately, an enhanced chest CT showed pulmonary masses became more larger compared with the former CT images in December 2018. EGFR 21 exon p.L858R mutation (1.15\%) was detect in bloodbased genetic test, so the patient took Gefitinib (Iressa, $0.25 \mathrm{~g}$, qd) instead of Anlotinib form January 2019 to March 2019.

The disease progressed with lager pulmonary masses and more pleural effusion in April 2019. The patient received 2 cycles of chemotherapy with albumin-bound Paclitaxel plus Carboplatin and Bevacizumab (albumin-bound Paclitaxel 300 mg/cycle, Carboplatin 400 mg/cycle, Bevacizumab $440 \mathrm{mg} /$ cycle, $3 \mathrm{w} /$ cycle). It came up with IV ${ }^{\circ}$ myelosuppression. As a result, chemotherapy protocols was changed with albumin-bound Paclitaxel plus Cisplatin and Bevacizumab (albumin-bound Paclitaxel $200 \mathrm{mgg} /$ cycle, Cisplatin $400 \mathrm{mg} /$ cycle, Bevacizumab $440 \mathrm{mg} /$ cycle, $3 \mathrm{w} /$ cycle). The patient was received 4 cycles of above chemotherapy.

The patient's condition progressed with brain metastases in December 2019, EGFR 21 exon p.L858R mutation (1.15\%) was detect in blood-based genetic test, so the patient received 5 cycles of Erbtinib (Tarceva) with bevizumab (Erbtinib 150 mg qd, Bevizumab 450 mg, q3w).

The patient found a mass in the left breast by herself-examination in June 2020. Mammograms revealed Multiple mass in the left breast, BI-RADS 4B (Fig. 1). Therefore, breast and lymph node puncture in ultrasound-guided were performed and the postoperative pathological diagnosis of metastatic lung adenocarcinoma was confirmed (Fig. 2). The patient received pembrolizumab (Keytruda) (220 mg q3w). We recorded the results of the tumor biomarkers before the patient's each treatment (Table 1).

Fig 1 Mammograms Multiple mass in the left breast, BI-RADS 4B

Immunohistopathological analysis demonstrated negative stain for ER(B), PR(C),Gata-3(D), GCDFP-15(E) and postive for CK7(F),TTF1(G),NapsinA(H) (orginial magnification $\times 400$ ). 
Table 1

The results of the tumor biomarkers before the patient's each treatment

\begin{tabular}{|lllll|}
\hline Date & CEA(ng/ml) & CA199(U/mL) & CA125(U/mL) & CA153(U/mL) \\
\hline $06 / 11 / 2020$ & 132 & 74.5 & 1314 & 116 \\
\hline $07 / 08 / 2020$ & 11.8 & 30.5 & 171 & 117 \\
\hline $07 / 29 / 2020$ & 15.5 & 21.8 & 81.7 & 76.7 \\
\hline $08 / 25 / 2020$ & 16.9 & 1544 & 107 & 85.4 \\
\hline $09 / 22 / 2020$ & 26.3 & 84.9 & 122 & 79.8 \\
\hline $10 / 05 / 2020$ & 33.2 & 133 & 177 & 108 \\
\hline $11 / 23 / 2020$ & 35.4 & 9370 & 602 & 69.5 \\
\hline
\end{tabular}

However, disease progressed again with larger brain metastatic lesions in August 2020.The maximum diameter of the patient's breast mass did not change significantly before and after immunotherapy (Fig. 3). The patient underwent genetic testing again. The test results are the same as before. Gene detection of breast metastasis showed EGFR 21 exon p.L858R mutation. The patient received Almonertinib (110 mg po qd). After the patient has used Almonertinibfor one month, we can clearly see from the head MRI that the patient's lesions are smaller than before (Fig. 4). And the tumor biomarkers were significantly lower than before. The patient's breast lesions have been in a stable state. The patient's condition was partially relieved. Later patients have maintained oral Almonertinib targeted therapy until now. The patient's condition is currently in a stable state.

\section{Discussion}

There are big differences between primary breast cancer and secondary breast cancer in clinical features, imaging, pathology and immunohistochemistry. This patient started with pericardial effusion and pleural effusion. Breast masses are clues. Adenocarcinoma cells were found through puncture and drainage of pericardial effusion and exfoliated cytology. Combined with immunohistochemistry, tumorous lesions in the lung were further found. At the time of diagnosis, it was at an advanced stage. It is easy to be considered as primary breast cancer at the first diagnosis, or the coexistence of lung cancer and breast cancer. The role of histopathology as the gold standard in the diagnosis of diseases is beyond doubt. However, the morphological identification of small biopsy specimens with atypical morphology is limited, and immunohistochemical testing is indispensable for diagnosis at this time. At present, the commonly used immune markers in lung adenocarcinoma include NapsinA, TTF-1, CK7 and alveolar surface glycoprotein, etc ${ }^{6}$. Breast cancer mainly expresses ER/PR, GATA-3 and Mamma-globin, etc. The positive rate of GATA-3 is $47 \%-100 \%$, which is a specific marker of breast cancer ${ }^{7}$. Immunohistochemistry of the breast tumor in this patient showed positive TTF-1, NapsinA, and CK7, supporting the diagnosis of lung adenocarcinoma. The patient's immunohistochemical results showed TTF-1 positive and ER negative. Although a small number of breast cancers can also express TTF-1, they need to be distinguished from 
primary breast cancer ${ }^{8,9}$. Because of the more specific NapsinA positive and GATA-3 negative, the diagnosis of lung adenocarcinoma and breast metastasis is clear.

Primary malignant tumor of breast is one of the most common malignant tumors in adult women, but as the breast is rich in fibrous tissue, poor blood circulation, so secondary metastasis of breast is very rare. Metastatic breast tumors are very rare, accounting for only $0.4-1.3 \%$ of breast malignancies, and the most common are leukemia, lymphoma, melanoma, rhabdomyosarcoma, and lung cancer ${ }^{10}$. The ways of breast metastasis in patients with primary lung cancer are as follows: To the ipsilateral breast through intrathoracic lymphatic metastasis; Tumor cells along with the lymph circulation through the thoracic duct into the vein, through systemic circulation to the contralateral breast; Tumor cells enter the blood circulation and metastasize far away ${ }^{11}$.

Our patient had metastasis to her right breast, which is the same side affected by the malignant pleural effusion, consistent with the hypothesis by Huang et $\mathrm{al}^{12}$. To this end, they considered a stepwise mechanism involving parietal pleural seeding, followed by invasion into chest wall lymphatic vessels draining to ipsilateral axillary lymph nodes and retrograde lymphatic spreading to the breast. This mechanism of breast metastasis could be supported by findings of enlarged homolateral axillary lymph nodes. Moreover, Barber et $\mathrm{al}^{13}$ demonstrated lymphatic communication between the breast and mediastinal lymphatic channels. These hypotheses could be confirmed by the fact that almost $80 \%$ of the cases reported from 2000 to date had ipsilateral lesions. Another potential type of spread could be hematogenous. However, if lung cancer spreads through this route, both breasts should have the same probability of being affected. This is not reflected in the reviewed cases, where only $5.4 \%$ of patients had bilateral breast involvement. The last possible explanation could be direct tumor invasion through the chest wall to the breast, but chest CT scans did not reveal this alteration in the reported cases. Therefore, lymphatic spreading might be the most reasonable mechanism of lung cancer dissemination to the breast.

Mirrielees et al. analyzed through a systematic retrospective analysis of 43 cases of lung cancer breast metastasis reported from 1989 to 2013; Among them, 10 cases were small cell lung cancer, 33 cases were non-small cell carcinoma, including 19 cases of adenocarcinoma, 3 cases of squamous cell carcinoma, 4 cases of large cell carcinoma, 4 cases of neuroendocrine carcinoma, 3 cases of undifferentiated carcinoma or others; The report involved 38 female and 5 male patients, with similar incidence in each age group. Among them, the average age of breast metastasis in NSCLC patients was 55 years old, SCLC patients were 58 years old, and patients were between 28 and 83 years old ${ }^{14}$. The correlation between the incidence of lung cancer and breast metastasis and pathological types remains to be studied with large samples.

In general, patients with solid tumors that have metastasized to the breast already have extensive metastases in other locations, and most patients survive for no more than one year after breast metastasis is found ${ }^{15}$. In a study of 169 patients with advanced solid tumors of different types of nonbreast origin, the median survival time of patients after diagnosis of breast metastasis was 10 months (1 
to 192 months) ${ }^{16}$. At present, there is no clear guideline for patients with breast metastasis, and the management and treatment are mainly based on the pathology, symptoms, stage, and physical condition of the primary malignant tumor ${ }^{15}$. In general, radical surgery is not recommended in patients with metastatic cancer and may lead to higher complications and mortality ${ }^{15}$. According to the latest ESMO guidelines, patients with solitary metastasis of lung adenocarcinoma can consider radical treatment of primary and metastatic lesions on the basis of systemic therapy, while patients with multiple metastases of lung adenocarcinoma should be given systemic therapy ${ }^{17}$.

Our case was advanced lung cancer, genetic testing revealed an EGFR mutation, and Almonertinib was treated. Almonertinibis the second third-generation EGFR-TKI innovative drug in the world. It is mainly used for disease progression during or after treatment with EGFR TKI. The test confirmed the presence of EGFR T790M mutation-positive adult patients with locally advanced or metastatic non-small cell lung cancer. Almonertinibis easier to cross the blood-brain barrier than other EGFR-TKI targeted drugs. We took into account the presence of brain metastases in our patient, so in the case of ineffective immunotherapy, we chose amitinib targeted systemic therapy. As a result, the patient controlled the brain lesions and achieved stable disease control for 2 months. Treatment is currently underway. We will continue to track and record the patient's disease.

\section{Conclusion}

In conclusion, although breast metastasis from lung cancer is rare. An accurate diagnosis is of great clinical importance. Distinguishing whether breast malignant tumors are primary or secondary is directly related to the principles of treatment and prognosis. For lung cancer patients with recurrent pleural effusion, visit of the breast should be included in the follow-up process. At present, there are few clinical cases, and it is necessary to accumulate clinical time to specify the best treatment plan.

\section{Abbreviations}

CT: computed tomography, NSCLC: non-small cell lung cancer, EGFR: epidermalgrowth factor receptor, TKI: tyrosine kinase inhibitor, CK 7: cytokeratin 7; TTF1: thyroid transcription factor 1, MRI: Magnetic Resonance Imaging.

\section{Declarations}

\section{Acknowledgements}

The authors thank to all members of the study team, the patient and her family.

\section{Authors's contributions}

Chanchan Gao was responsible of the clinical management of the patient presented and acquisition of data, and helped to draft the manuscript. Xuyu Gu and Shiya Zheng were responsible of the clinical 
management, interpretation of the data, and drafting the manuscript. Longfei Wang collected and analyzed data. The author(s) read and approved the final manuscript.

\section{Funding}

Supported by the Foundation Research Project of Jiangsu Province the Natural Science Youth Fund NO. BK20200395 and Jiangsu Provincial Health Commission H2019107.

\section{Availability of data and materials}

Data sharing is not applicable to this article as no datasets were generated or analysed during the current study.

\section{Ethics approval and consent to participate}

The study was approved by Zhongda Hospital Southeast Universtiy. The patients consented to participate.

\section{Consent for publication}

Written informed consent for research and publication from the patients was obtained.

\section{Competing interests}

The authors declare that they have no competing interests.

\section{Author details}

${ }^{1}$ School of Medicine, Southeast University, Nanjing 210009, P.R. China; ${ }^{2}$ Department of oncology, Zhongda Hospital, School of Medicine, Southeast University, Nanjing 210009, P.R. China; ${ }^{3}$ Department of pathology, Zhongda Hospital,School of Medicine, Southeast University, Nanjing 210009, P.R. China; \# Corresponding author: Shiya Zheng phD, Tel:+8615805160130, E-mail: zhengshiya1990@163.com.

\section{References}

1. Bade BC, Dela Cruz CS. Lung Cancer 2020: Epidemiology, Etiology, and Prevention. Clin Chest Med. 2020;41(1):1-24

2. Wu Z, Yuan Q, Yang C et al. Downregulation of oncogenic gene TGF $\beta R 2$ by miRNA-107 suppresses non-small cell lung cancer. Pathology, research and practice. 2020;216(1):152690

3. Huang $X$, Zhu L, Zhou L et al. Multidisciplinary and Comprehensive Chinese Medicine for Advanced Non-Small Cell Lung Cancer Patients: A Retrospective Study of 855 Cases. Chin J Integr Med. 2020;

4. Hagimoto S, Miyazaki K, Sato S, Kodama T, Satoh H. An autopsied NSCLC patient with huge adrenal gland metastasis with rapid growth and local invasion. Tuberkuloz ve toraks. 2019;67(2):142-145 
5. Georgiannos SN, Chin J, Goode AW, Sheaff M. Secondary neoplasms of the breast: a survey of the 20th Century. Cancer-Am Cancer Soc. 2001;92(9):2259-66

6. Gurda GT, Zhang L, Wang Y et al. Utility of five commonly used immunohistochemical markers TTF1, Napsin A, CK7, CK5/6 and P63 in primary and metastatic adenocarcinoma and squamous cell carcinoma of the lung: a retrospective study of 246 fine needle aspiration cases. Clinical and translational medicine. 2015;4:16

7. El Hag MI, Ha J, Farag R, El Hag AM, Michael CW. Utility of GATA-3 in the work-Up of breast adenocarcinoma and its differential diagnosis in serous effusions:: A Cell-Block Microarray Study. Diagn Cytopathol. 2016;44(9):731-6

8. Ni Y, Tsang JYS, Shao M et al. TTF-1 expression in breast carcinoma: an unusual but real phenomenon. Histopathology. 2014;64(4):504-11

9. Voutsadakis IA, Mozarowski P. Expression of TTF-1 in breast cancer independently of ER expression: A case report and pathogenic implications. Breast disease. 2017;37(1):1-6

10. Copur MS, Lackner R, Rodriguez P et al. Recurrent EGFR-Mutated Non-Small Cell Lung Cancer Discovered by Abnormal Mammogram: Adjuvant/Frontline Metastatic Management Options. Oncology (Williston Park, N.Y.). 2020;34(9):347-351

11. Huang $\mathrm{H}$, Hang J, Wu M, Chou T, Chiu C. Lung adenocarcinoma with ipsilateral breast metastasis: a simple coincidence? Journal of thoracic oncology : official publication of the International Association for the Study of Lung Cancer. 2013;8(7):974-9

12. Huang $\mathrm{H}$, Hang $\mathrm{J}$, Wu $\mathrm{M}$, Chou $\mathrm{T}$, Chiu $\mathrm{C}$. Lung adenocarcinoma with ipsilateral breast metastasis: a simple coincidence? Journal of thoracic oncology : official publication of the International Association for the Study of Lung Cancer. 2013;8(7):974-9

13. Barber TW, Hofman MS, Hicks RJ. Breast lymphatic drainage via the pulmonary lymphatic system. Eur J Nucl Med Mol I. 2010;37(11):2203

14. Mirrielees JA, Kapur JH, Szalkucki LM et al. Metastasis of primary lung carcinoma to the breast: a systematic review of the literature. The Journal of surgical research. 2014;188(2):419-31

15. Ninan J, Naik V, George GM. 'Inflammatory breast cancer' due to metastatic adenocarcinoma of lung. BMJ case reports. 2016;2016

16. Mirrielees JA, Kapur JH, Szalkucki LM et al. Metastasis of primary lung carcinoma to the breast: a systematic review of the literature. The Journal of surgical research. 2014;188(2):419-31

17. Planchard D, Popat $S$, Kerr $\mathrm{K}$ et al. Metastatic non-small cell lung cancer: ESMO Clinical Practice Guidelines for diagnosis, treatment and follow-up. Annals of oncology : official journal of the European Society for Medical Oncology. 2018;29(Suppl 4):iv192-iv237

\section{Figures}






\section{Figure 1}

Mammograms Multiple mass in the left breast, BI-RADS 4B
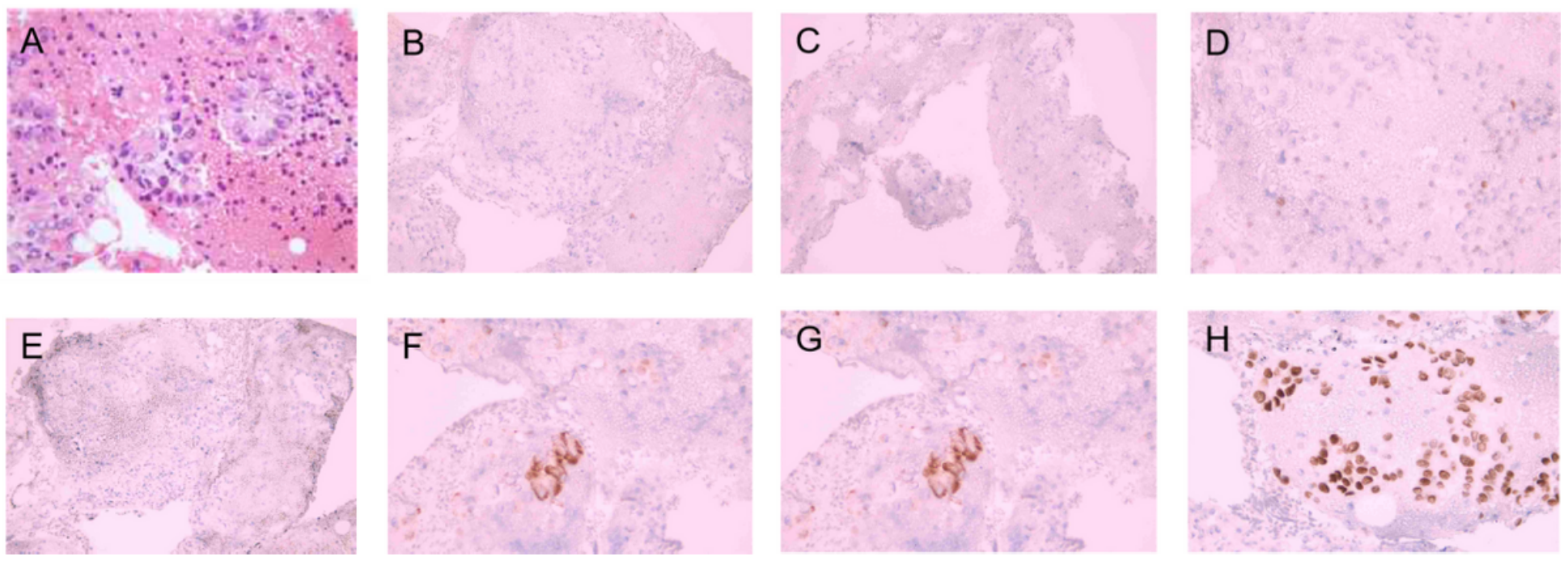

Figure 2

FNA cell block in left axillary lymph node showed adenocarcinoma cells:hematoxylin and eosin stain(A) (orginial magnification $\times 200$ ). 

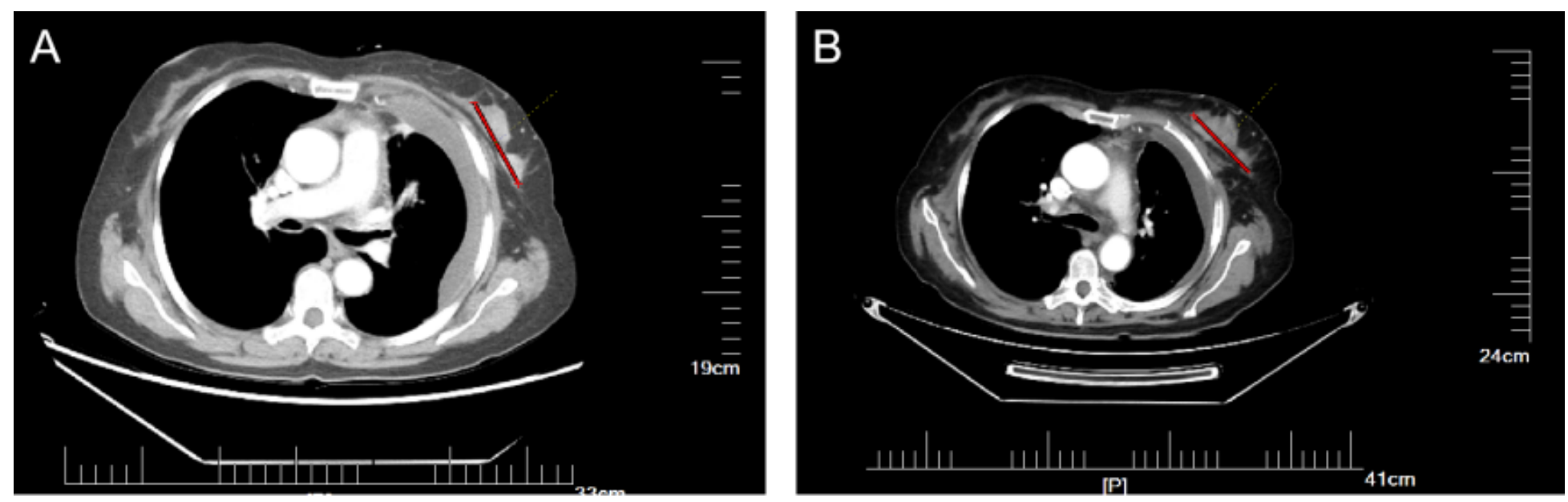

Figure 3

Chest computed tomography (CT)
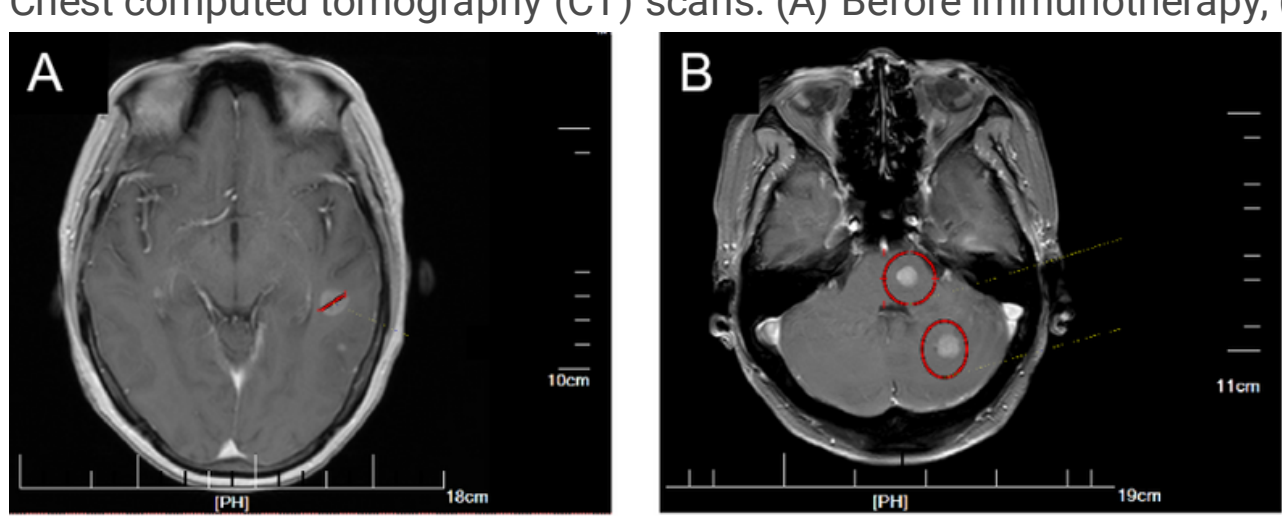

(B)After immunotherapy.



Figure 4

Head magnetic resonance imaging (MRI): (A)Before immunotherapy, (B) After immunotherapy, (C)After targeted therapy. 